\title{
RF CONTROL SYSTEM UPGRADE AT CAMD
}

\author{
D Launey, M Fedurin, P Jines, V Suller, CAMD, Baton Rouge, LA, USA
}

\section{Abstract}

A description is given of the new control system for the RF system of the CAMD light source. The new design being implemented brings all RF signals into the data acquisition system via a modular, custom made, RF detector and renders the amplitude and tune control loops in the VME computer. On line calculations ensure monitoring of proper operation and display the information to the user in an efficient way. In addition, an advanced load impedance monitoring diagnostic has been implemented, being displayed as a Smith Chart, which is based on the system used at the SRS in Daresbury, England.

\section{INTRODUCTION OF RF SYSTEM}

The CAMD (Center for Advanced Microstructures and Devices) RF system comprises of a $60 \mathrm{~kW}$ klystron, LBand waveguides, circulator, adjustable sliding short and DORIS type cavity. The tuning plunger is activated by a stepper motor. Closed loop controls are comprised of an amplitude control loop and a phase control loop. The phase loop uses the phase difference between WGFP (WaveGuide Forward Power), a directional coupler before the sliding short and cavity, and the measuring loop in the cavity (See Figure 1) to adjust the plunger.

Though the implementation of the RF control system is robust and reliable since operational life of CAMD, there was very little diagnostics, both for the operator in the control room, and the RF Engineer. Reliable information about the RF has always been difficult to obtain, because the diagnostics were not always readily available and in place. The following paragraphs describe the newly implemented diagnostics system at CAMD and future improvements.

\section{LOAD IMPEDANCE DIAGNOSTIC}

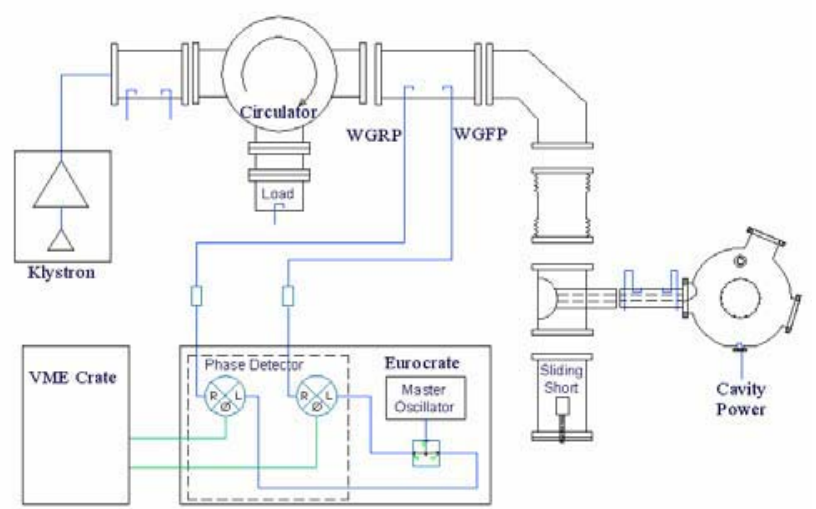

Figure 1: Load Impedance Diagnostic at CAMD.
A significant difficulty encountered with diagnosing problems with the RF system, and consequently, sometimes with the ring, was to determine the state of the RF system. Without a dedicated network analyzer, the load impedance of the cavity was not readily available. A relative inexpensive solution was to use a custom made, dedicated system used at the SRS Labs, in Daresbury, UK The system precisely measures the phase difference between, and amplitude of, two RF signals [1]. CAMD's implementation of the load impedance measuring system is shown in Figure 1. The phase difference is measured between the WGFP and the WGRP (WaveGuide Reverse Power) directional couplers, which determines the impedance characteristics of both the sliding short and the cavity as one load. The phase difference, along with the amplitudes of each RF signal, is delivered to ADCs in a VME crate. The software, written in Visual Basic and provided by SRS Labs, analyzes the measurements provided and displays a Smith Chart, complex load impedance, VSWR, detune angle, and reflection coefficient. Modifications to the software replaced SRS's backend control interface, with an interface with the EPICs system at CAMD. The results of the load impedance diagnostic after the system installation and initial calibration are presented in Figure 2.

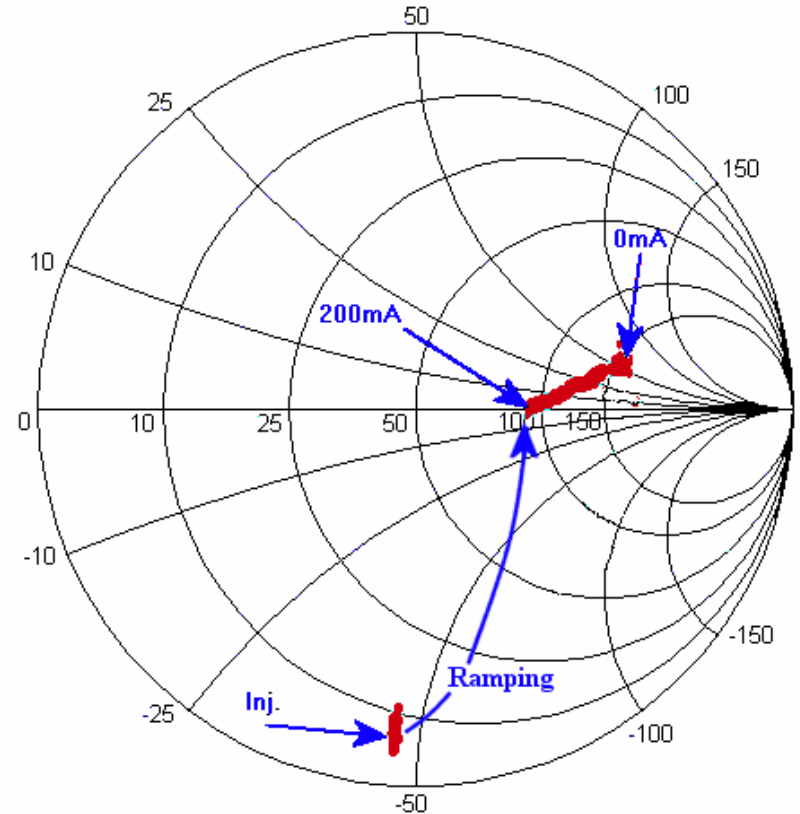

Figure 2: CAMD Smith Chart Results

At injection energy $(180 \mathrm{MeV})$, the load hovers near the 10 -j50 point, at the capacitive side of the resonance line. Once the ring is ramped to top energy $(1.3 \mathrm{GeV})$, the load moves near the $90+\mathrm{j} 0$ point, assuming that the beam current is about $200 \mathrm{~mA}$. As the beam decays to $0 \mathrm{~mA}$, the load moves to $150+\mathrm{j} 50$ point. Note that, this load is the 
cavity, sliding short, and the beam loading. Therefore, moving to the inductive side of resonance will be observed. Regarding the Robinson instability and the resonance of the cavity, the beam drops when the load on the Smith Chart nears $50+\mathrm{j} 75$.

\section{RF DIAGNOSTIC MONITORING SYSTEM}

\section{Hardware Implementation}

An inexpensive RF detector device developed in house monitors the various RF powers in the RF system. The design is based on the AD8361 power detector, an IC commonly used to measure the power levels in cell phones. The circuit is mounted in a small $(2 \times 1.5 \times 1.25$ $\mathrm{cm})$ aluminum modified enclosure with connectors for DC power, RF Input, and detector output. This allows for easy replacement in case of failure. A numerical LED display mounted on the front displays the voltage of the detector output. Figure 3 shows the detector calibrations for RF input in volts RMS and detector output in volts. The output is linear and very consistent.

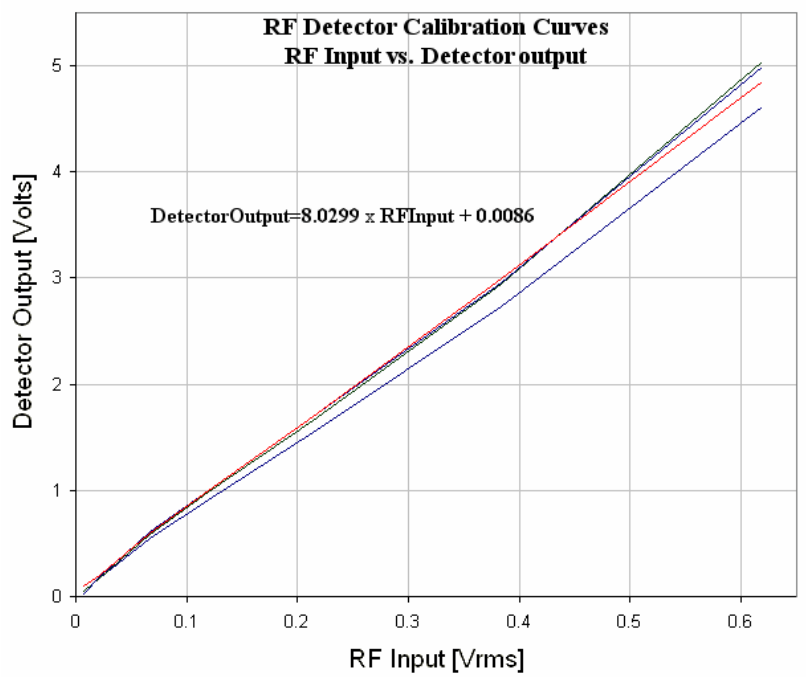

Figure 3: RF Detector Calibration Curves

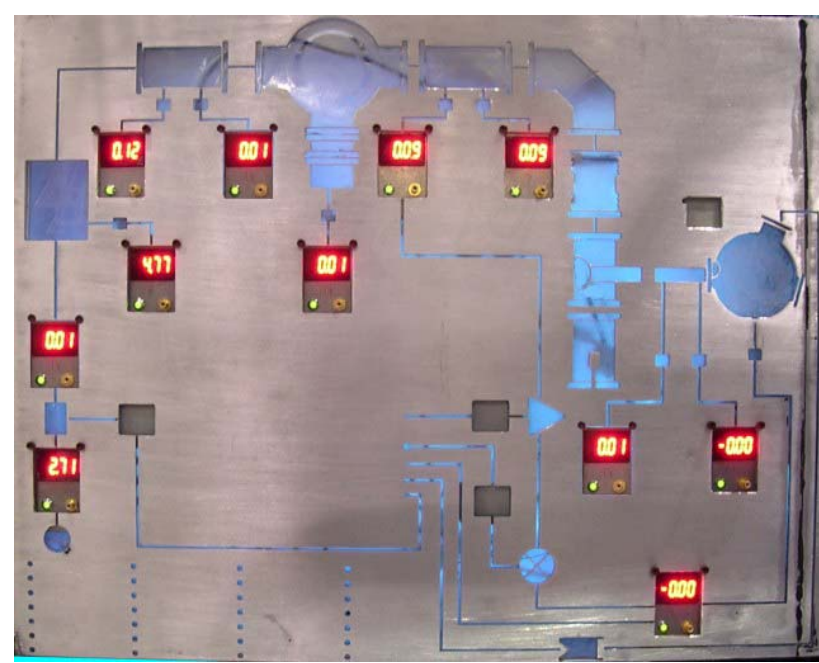

Figure 4: Local Display Panel for RF Powers
These RF detector modules are mounted on a panel near the klystron and positioned to follow a schematic representing the RF system and the various signals, as seen in Figure 4. The 0-5 volt output of the RF detectors is brought to ADCs in a VME crate.

\section{Software Implementation}

With the recent installation of the VME hardware and EPICs control system at CAMD [2], a custom graphical user interface was created in house to display all parameters related to the RF System. (see Figure 5).

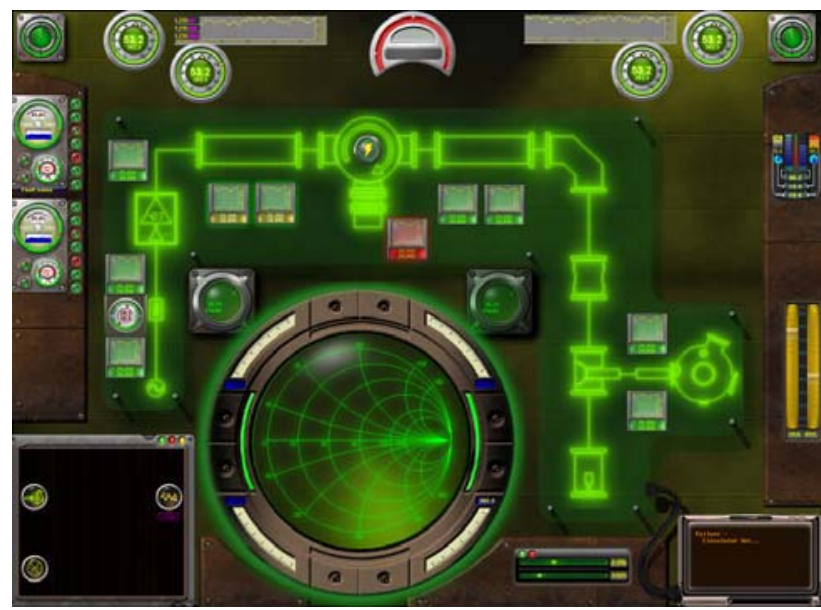

Figure 5: Dedicated Graphical Display of the RF System

The real time, always on, display shows the status of the RF system on a dedicated monitor in the control room. The software is integrated with the modified version of the load impedance diagnostic software for a complete and thorough diagnostic display of the RF system for both the ring operator and the RF Engineer. The display presents the diagnostic information efficiently and effectively as a, stylistically designed, schematic of the $\mathrm{RF}$ system. Because the ring operator is not necessarily concerned with the intimate details of the RF system, colors indicate good condition (green) or fail condition (red) for each parameter in the RF system. Using the same display, the RF Engineer quickly and accurately pin point faults in the system. For example, if the beam current drops out, the operator can first glance at this display to see any red and determine if the RF is responsible for the failure. Furthermore, the RF Engineer can use the display to confirm if the various system conditions are within specifications. To aid the RF Engineer, most RF power values are presented in kilovolts, kilowatts, and $\mathrm{dBm}$ (based on a $50 \mathrm{ohm}$ impedance measuring system). Approximately 150 unique parameters are displayed on one screen at once. Other examples from the display include real time display of klystron gain, pre-amp gain, klystron perveance, circulator insertion loss, circulator effectiveness and efficiency, theoretical system power losses, measurements for the control loop components, and, of course, alarms 
and faults. All parameters are being logged at a rate of $2 \mathrm{~Hz}$, and playback abilities will be implemented in the future. Data logging provides diagnostic features that did not exist until now, including improved recognition of trends and slowly occurring failures.

\section{PLANNED RF CONTROL SYSTEM}

Currently, at the beam current requirements, the RF system handily provides the needed RF power, although, it is near the upper limit. However, including the cavity losses, transmission losses, and beam loading, it is estimated that the system requires slightly more than $50 \mathrm{~kW}$ of RF power. Future plans at CAMD include the installation of a second insertion device. This will require a second RF cavity, utilizing our spare cavity, spare circulator, and spare klystron. Currently, the control system for the RF is rendered solely by custom made electronics. However, a new control system will utilize the newly installed RF detector modules and the actual control loops will be rendered in the VME crate controller. Preliminary tests indicate that this is a feasible solution. This solution provides more diagnostic tools, such as automated plunger scanning to determine the resonance curve of the cavity. It also improves the current control system to provide more flexibility and control, such as manual, or open loop, plunger control and amplitude control.

\section{CONCLUSION}

In addition to the WGFP and WGRP signals in the load impedance diagnostic, future improvements could include adding the signals from the directional couplers just before the cavity, and after the sliding short coupling. This will provide the impedance of the cavity itself without including the sliding short as part of the load. This will more accurately give information to what the electron beam sees, not at what the klystron's load is.

Although the numerical LED displays on the RF detectors are very useful, subsequent revisions of the detectors will implement a separate enclosure for the displays.

With the addition of the described diagnostics enhancements, CAMD now has real time, always on, information about the RF system that was previously unavailable, or not readily available. Furthermore, the logging of RF data facilitates process control trending and comparison to historical data. The new diagnostic tools significantly improve the identification and resolution of problems and easily provide RF system performance as it relates to ring operation.

\section{REFERENCES}

[1] CW Horrabin; "A $500 \mathrm{MHz}$ Mixer-Type Phase Detector with Wide Dynamic Range and Small Phase Error", Proc EPAC'94, pp1975-1977

[2] VP Suller, M Fedurin, P Jines, D Launey, T Miller and Y Wang; "Improvements To, And Current Status Of, The CAMD Light Source," Proc EPAC'04, pp2424-2426 